\title{
A study of analysis method for the identification of UHECR source type
}

\author{
Fugo Yoshida, ${ }^{a, *}$ Yuichiro Tameda ${ }^{a}$ and for the Telescope Array Collaboration \\ ${ }^{a}$ Department of Engineering Science, Faculty of Engineering Osaka Electro-Communication University \\ 18-8, Hatsucho, Neyagawa-shi, Osaka, JAPAN \\ E-mail: me20a014@oecu.jp, tameda@osakac.ac.jp
}

The autocorrelation analysis using the arrival direction of Ultra High Energy Cosmic Rays (UHECRs) has been previously reported by the Telescope Array (TA) experiment. It is expected that the autocorrelation function reflects the source distribution. We simulate the expected arrival direction distribution of the cosmic rays using the catalogs of candidate sources. We take into account random deflection in the magnetic fields, with the magnitude of deflection determined by the charge and energy of the cosmic rays, coherence length and magnitude of the extragalactic magnetic field (EGMF), and by distance to source. In addition, in order to compare with the results of TA, we consider the TA exposure. We compare the autocorrelation of the arrival directions corresponding to different source catalogs with the isotropic distribution. We calculate the autocorrelation function for each type of source candidates using this procedure. We will discuss the ability of this method to identify the source type of UHECRs.

$37^{\text {th }}$ International Cosmic Ray Conference (ICRC 2021)

July 12 th - 23rd, 2021

Online - Berlin, Germany

\footnotetext{
${ }^{*}$ Presenter
} 


\section{Introduction}

I am investigating the origin-dependence of the clustering of cosmic ray arrival directions observed by the Telescope Array (TA) experiment. Since cosmic rays are charged, their direction of motion is bent by the magnetic field in space and they lose their directionality. Therefore, the origin of cosmic rays arriving at the earth has not been clarified yet, and it is considered to be an important issue in the field of astrophysics. On the other hand, UHECRs which arrive at a frequency of about one per $1 \mathrm{~km}^{2}$ per year, are less affected by magnetic fields and travel almost straight from their origin. Therefore, the TA experiment aims to find a clue to the origin of UHECRs by observing them with two types of detectors, Surface Detector (SD) and Fluorescence Detector (FD), installed in Utah, USA, since 2008 [1]. The TA experiment has so far captured signs of a high concentration of UHECRs from a certain direction [2]. However, the frequency of the arrival of UHECRs to the earth is very small, so we need to increase the statistics from long-term observations. In addition, the TA $\times 4$ experiment has partially started in 2019, enabling higher statistics observations [3]. Therefore, I decided to search for clues to the origin of the cosmic rays by using an analysis method that does not depend on the observation statistics using the current observation data. First of all, I focused on the clustering excess in the direction of arrival of the events observed by the TA experiment, and assumed that the distribution of the origin is reflected in the result. This is because if multiple cosmic ray emitting origins are isotropically distributed, we would expect to find no clustering excess in the events observed on Earth. Therefore, in this analysis, we generate cosmic ray events arriving at the Earth from a group of candidate origins by Monte Carlo (MC) simulations and investigate their clustering excess. If the simulation results reproduce the results of previous studies, we can identify or narrow down the origin group.

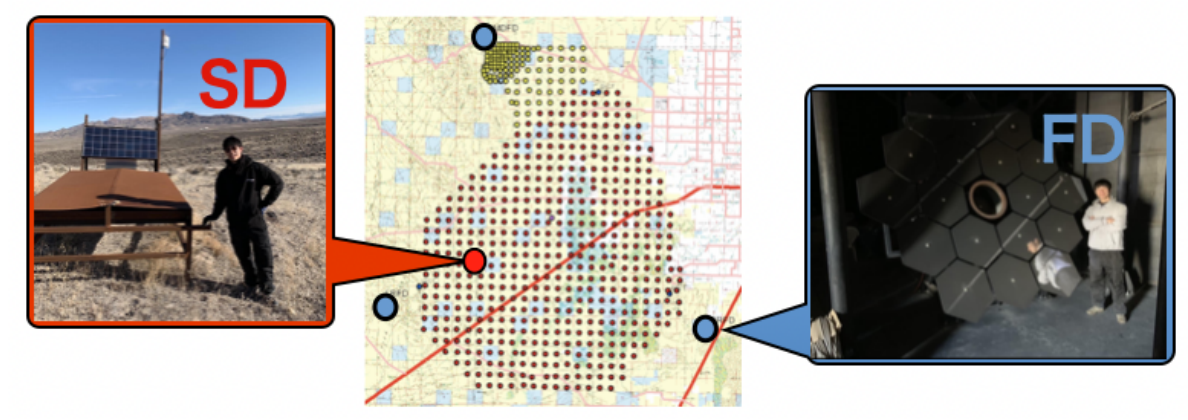

Figure 1: Detector configuration map of the Telescope array (TA) experiment. The red circles are 507 surface particle detectors (SD) spaced $1.2 \mathrm{~km}$ apart. The SDs are surrounded by three atmospheric fluorescence telescopes (FD), indicated by blue circles. The northernmost FD is called Middle Drum (MD), and the southern FD is called Black Rock Mesa (BR) and Long Ridge (LR). The TA experiment realizes a hybrid observation of FD and SD. The yellow circles around MD indicate the TALE SD.

\section{Autocorrelation Function}

The clustering of CR events at a given angular scale appears in the autocorrelation function of the events [4][5][6]. The autocorrelation function is determined by the excess of pairs of events 
separated by a given angular distance $\delta$ as compared to a uniformly distributed set with the same total number of events. The procedure is as follows: for a given separation angle $\psi$, we count the number of pairs of data events that are separated by an angular distance $\delta$ less than $\psi$. Next, we generate a large number of uniformly distributed MC event sets that take into account TA exposure with the same number of events as the real dataset. In each MC set we count pairs of events in the same way as in the data,which gives the MC count for that set. For each value of $\psi$ we then determine the fraction of simulated sets where the number of pairs is greater than or equal to the number of pairs in the data. This gives the $\mathrm{P}(\psi)$. When the value of $\mathrm{P}(\psi)$ is small, it means that the number of pairs is excessive (not uniform) at a certain angular scale. The clustering of UHECR events at $\mathrm{E}>57 \mathrm{EeV}$ observed by the TA experiment (Fig. 2) shows the largest excess at distances between the events of about $20^{\circ}$ and $30^{\circ}$. I thought that this autocorrelation function would reflect the distribution of the source. Therefore, we came up with the idea we may be able to identify the source type from the characteristics of the autocorrelation function assuming the arrival direction from source.

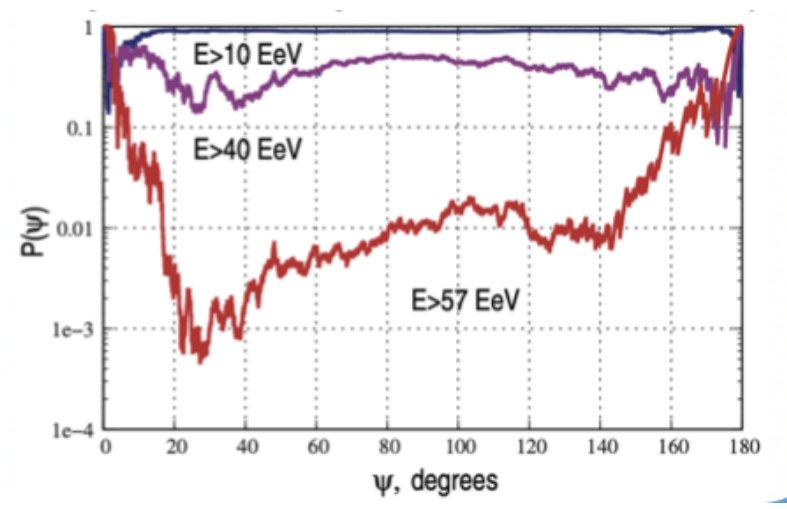

Figure 2: The results show the excess clustering of events at each energy threshold detected in the TA experiment. The horizontal axis is the Separation angle $\psi$, and the vertical axis is the degree of clustering excess in the direction of cosmic ray arrival, $\mathrm{P}(\psi)$. This result uses events obtained from 7 years of TA observations: 2996 events for $\mathrm{E}>10 \mathrm{EeV}, 210$ events for $\mathrm{E}>40 \mathrm{EeV}$, and 83 events for $\mathrm{E}>57 \mathrm{EeV}$. It shows that the clustering of events is isotropic when the energy threshold is low.

\subsection{UHECR source candidates}

We use the list of radio galaxies (RGs), which are the leading candidates for the origin of UHECRs presented at ICRC2019 [7], to simulate cosmic rays arriving from them. The RGs list contains 42 sources with five parameters: RA, Dec, $P_{1}, d$, and type. where $P_{1} / J_{y}$ is radio flux of the steep spectrum component of the source at $1 \mathrm{GHz}, \mathrm{d} / \mathrm{Mpc}$ represents the distance to the source. There are five types of RGs in the list: FR-1, FR-2, SSRQ, BLU, and BLO. we simulate the cosmic rays coming from these 42 radio galaxies, but it is unlikely that cosmic rays arrive with uniform probability from 42 radio galaxies, we determined the fraction of events arriving from radio galaxies based on the ratio of $\mathrm{P}_{1}$ to the cosmic ray flux $\mathrm{F}_{\mathrm{CR}}$ arriving at Earth from celestial objects.

$$
\mathrm{F}_{\mathrm{CR}}=\frac{1}{4 \pi \mathrm{d}^{2}}\left(4 \pi \mathrm{d}^{2} \mathrm{P}_{1}\right)^{\beta_{\mathrm{L}}}
$$


where $\beta_{L}$ is the relationship between the radio flux and the power of the jet is experimentally determined and is one of the parameters used to convert it to CR flux (for details, see [8]). In this analysis, $\beta_{L}$ uses 0.675 regardless of type.
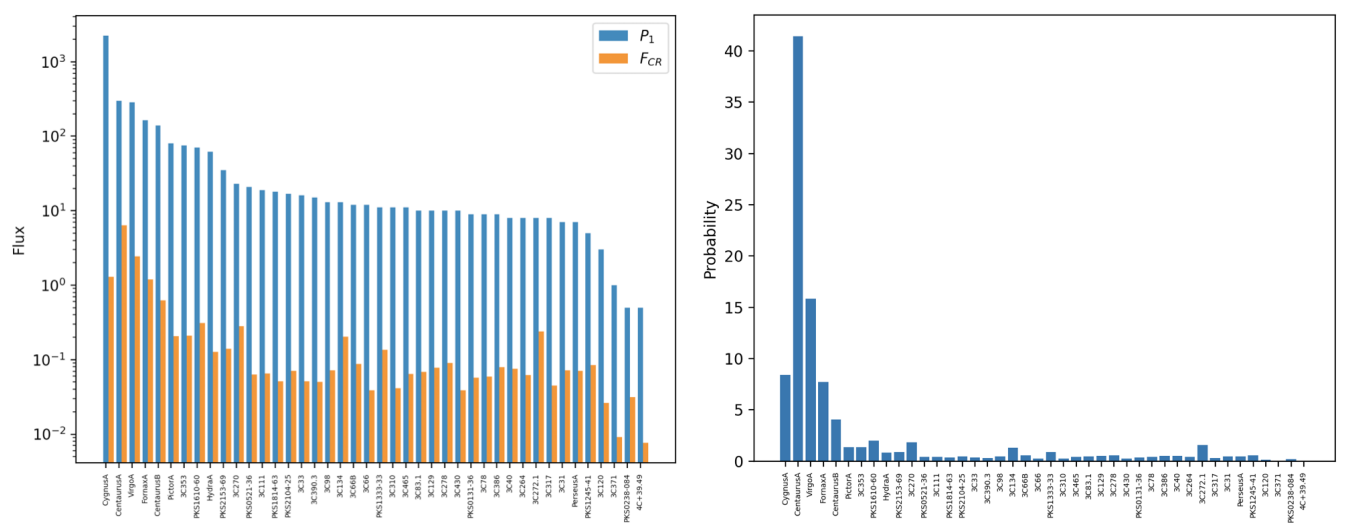

Figure 3: The left figure shows the relationship between the steep spectral component $P_{1}$ at $1 \mathrm{GHz}$ in the RGs list and the cosmic ray flux $F_{\mathrm{CR}}$ arriving at the Earth from the source obtained from equation 1 . The left figure shows the normalized cosmic ray flux $\mathrm{F}_{\mathrm{CR}}$ for each source and the number of events arriving at Earth from the source.

The left side of Fig. 3 shows the calculated $P_{1}$ and cosmic ray flux $F_{C R}$ for each source in the RGs list, and the right side of Fig. 3 shows the normalized cosmic ray flux $F_{C R}$ for each source and the number of events arriving at Earth from the source. 3C134 in the list is the source from which either protons or heavy nuclei of cosmic rays may originate and produce the most energetic air shower events, but its location behind the dense molecular cloud of our galaxy obscures the spectra needed to measure its redshift. The distance to $3 \mathrm{C} 134$ is estimated to be $30 \sim 300 \mathrm{Mpc}$ [9][10], we assumed $30 \mathrm{Mpc}$ (because $3 \mathrm{C} 134$ has a small radio flux, even assuming $300 \mathrm{Mpc}$ does not make much difference in the analysis results). From the figure, we can expect that Centaurus A, which is located in the southern hemisphere, will arrive at Earth with a probability of about $40 \%$, and Cygnus $\mathrm{A}$, which is located in the northern hemisphere, with a probability of $10 \%$. With these probabilities, we predict the number of events that will arrive on Earth from each source. The procedure for calculating the expected direction of arrival of cosmic rays from these sources is described next.

\subsection{Arrival direction of CRs expected from radio galaxies}

Since a magnetic field of a certain strength exists spatially in space, ultra-high energy cosmic rays are also bent in the propagation process. Therefore, it is necessary to consider the cumulative deflection angle of cosmic rays for each composition due to the magnetic field. The cumulative deflection of cosmic rays can be calculated theoretically from [11].

$$
\theta(E) \simeq 0.25^{\circ}\left(\frac{\mathrm{d}}{\lambda}\right)^{\frac{1}{2}}\left(\frac{\lambda}{1 \mathrm{Mpc}}\right)\left(\frac{\mathrm{B}}{10^{-9} \mathrm{G}}\right)\left(\frac{\mathrm{E}}{10^{20} \mathrm{eV}}\right)^{-1}
$$




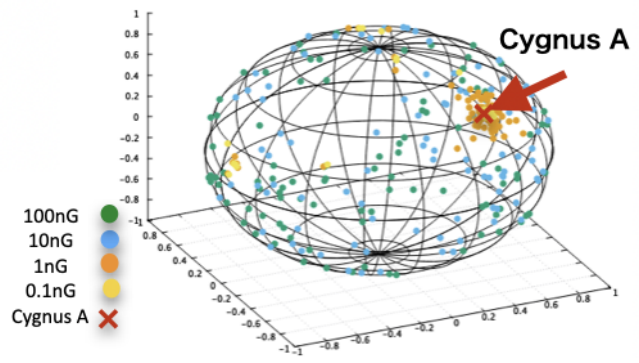

(a) Proton

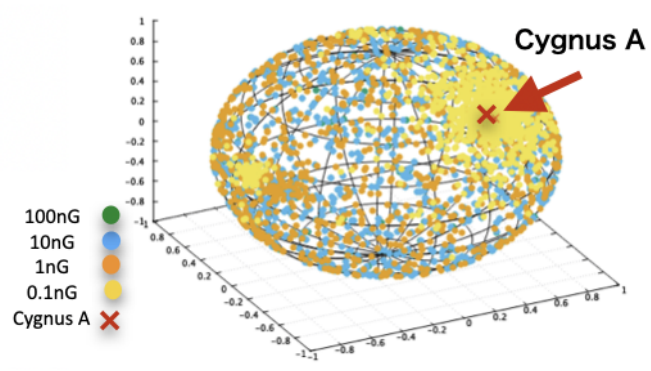

(b) Iron

Figure 4: This figure shows a stochastic reproduction of the degree of deflection of cosmic ray protons and iron arriving from Cygnus $\mathrm{A}$ using the equation 2 and the vMF function. The assumed magnetic fields are $10^{-7}, 10^{-8}, 10^{-9}$ and $10^{-10} \mathrm{G}$. The color points indicate the degree of scattering for each assumed magnetic field. The red points are for Cygnus A.

where $\mathrm{z}$ is the charge, $\mathrm{d}$ is the distance to the source, $\mathrm{B}$ is the magnetic field, $\lambda_{B}$ is the coherence length of the field, and $\mathrm{E}$ is the energy. If the EGMF is turbulent on a small scale, i.e., its coherence length $\lambda_{B}$ is smaller than the distance $\mathrm{d}$ to the source of the UHECR, it is expected to accumulate a series of small deflections stochastically. However, there is a problem that the deflection of UHECR cannot be determined in general, because the EGMF and coherence length are not yet understood. Constraints on the EGMF and coherence length have been advanced by [12][13]. Therefore, in this analysis, we assume three types of EGMF, $10^{-8}, 10^{-9}$, and $10^{-10}$, and assume that the EGMF is $1 \mathrm{Mpc}$ (controversial). Since cosmic rays do not always scatter at the same angle, but scatter stochastically, the degree of scattering in the direction of arrival is reproduced using the von Mises-Fisher (vMF) distribution, which is a two-dimensional Gaussian distribution on a sphere.

Fig. 4 shows the degree of scattering of cosmic ray proton and iron from Cygnus A in the RGs list. This figure shows the degree of scattering of cosmic ray protons and iron when propagating from Cygnus $\mathrm{A}(\mathrm{d}=250 \mathrm{Mpc})$ through magnetic fields $10^{-7}, 10^{-8}, 10^{-9}$ and $10^{-10} \mathrm{G}$. It can be seen that as the magnetic field becomes larger, the degree of scattering of cosmic rays also becomes more extensive. In addition, the iron has a larger charge than the proton, so it scatters over a wider area even when the magnetic field is small. Considering these assumptions, the directional distribution of proton and iron arrivals of cosmic rays expected from the RGs list is shown below.

Fig. 5 shows the distribution of cosmic ray arrival directions expected from radio galaxies assuming three types of magnetic fields and two types of compositions is shown in an equatorial coordinate system. The black stars indicate the positions of the radio galaxies, and the light blue points indicate the events expected from the radio galaxies. In order to compare the results with those of the TA experiment, we generated 150 events, the equivalent of the 10 years observed in the TA. The light blue points indicate the expected events from the radio galaxy, and these events are scattered within the field of view of the TA. The method of simulating the events in the field of view of the TA experiment is determined by the probability weighted by the exposure of the TA experiment from [14]. It is assumed that cosmic ray events from radio galaxies located at higher declination will be detected more frequently. A small number of events from outside the field of view of the TA experiment are also detected. As the assumed magnetic field becomes smaller, 


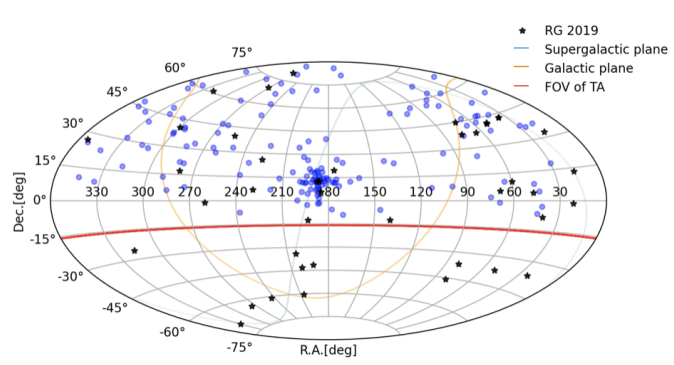

(a) Proton, $\mathrm{B}=10^{-8}$

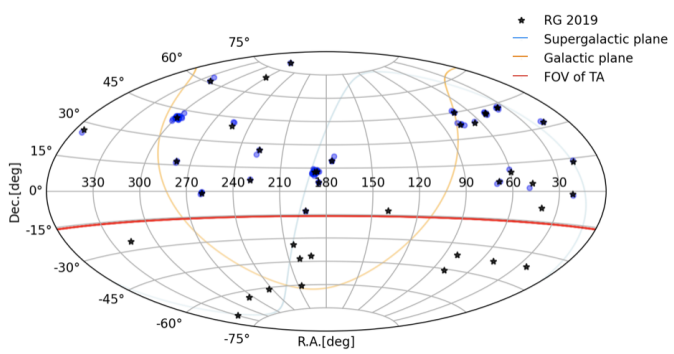

(c) Proton, $\mathrm{B}=10^{-10}$

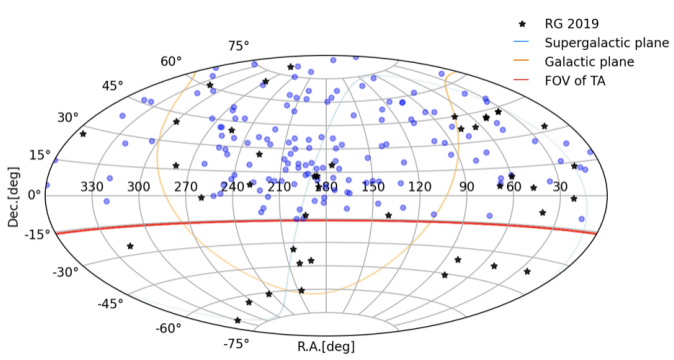

(e) Iron, $\mathrm{B}=10^{-9}$

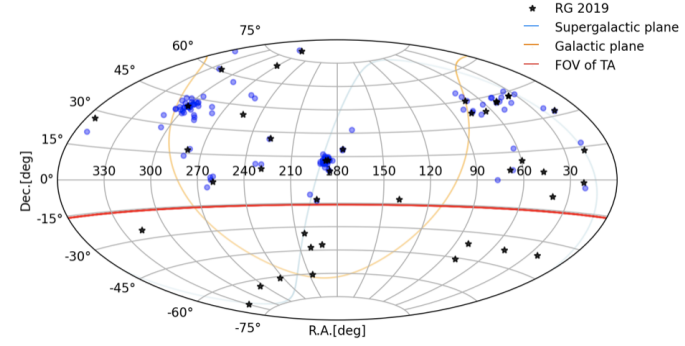

(b) Proton, $\mathrm{B}=10^{-9}$

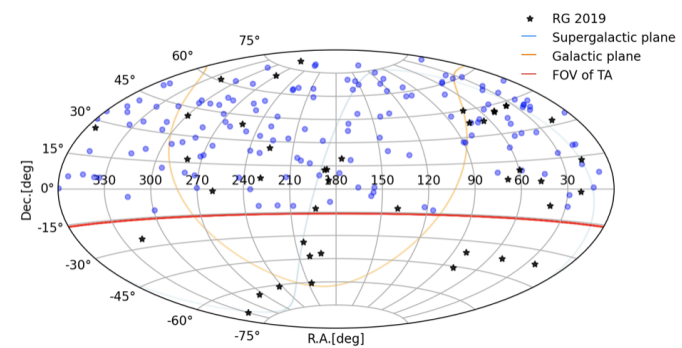

(d) Iron, $\mathrm{B}=10^{-8}$

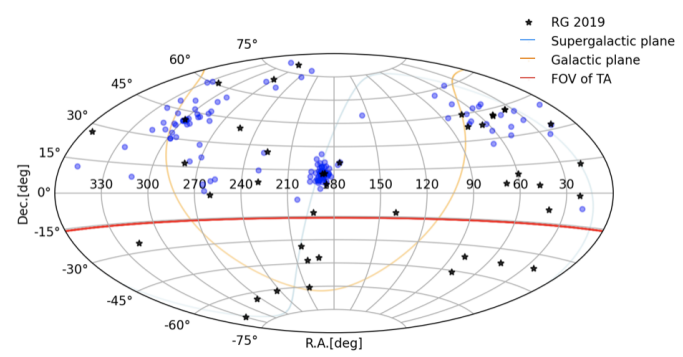

(f) Iron, $\mathrm{B}=10^{-10}$

Figure 5: The distribution of cosmic ray arrival directions expected from radio galaxies assuming three types of magnetic fields and two types of compositions is shown in an equatorial coordinate system. The black stars indicate the positions of the radio galaxies, and the light blue points indicate the events expected from the radio galaxies.In order to compare the results with those of the TA experiment, we generated 150 events, the equivalent of the 10 years observed in the TA.

more events are distributed around the point source of the source. This means that the deflection of cosmic rays from the source was small. Also, as the magnetic field increases, the deflection of the cosmic rays from the source is larger, which indicates that the events are distributed over a wider area. Comparing the results of the proton and iron simulations, the deflection of the cosmic rays is larger for iron due to its larger charge, indicating that the events are more widely distributed even in smaller magnetic fields.

The angular distance is calculated for each event in the arrival direction distribution generated by the MC simulation (non-duplicated). Then, the number of pairs of angular distances within a certain separation angle is counted, and $\mathrm{n}_{\text {corr }}^{\mathrm{MC}}$ is calculated. At the same time, the arrival direction distribution is generated from an isotropic MC simulation that takes into account the TA exposures, and $\mathrm{n}_{\mathrm{iso}}^{\mathrm{MC}}$ is calculated. From the comparison of the number of pairs of separation angle between 
these MC and the isotropic MC, the autocorrelation Function is calculated. The calculation method and results are shown below.

\section{Results}

Examine the correlation of arrival directions between $\mathrm{MC}$ events. The autocorrelation function is calculated by comparing $\mathrm{n}_{\text {iso }}^{\mathrm{MC}}$ and $\mathrm{n}_{\text {corr }}^{\mathrm{MC}}$. The distribution of $\mathrm{P}(\psi)$ is obtained by comparing the $10^{2}$ set of MC distributions with the $10^{5}$ set of isotropic MC distributions, since the distribution of arrival directions expected from the RGs list varies from simulation to simulation even under the same conditions. For each separation angle $\psi$, determine the median $\mathrm{P}_{\text {med }}$ of the distribution and the $68 \%$ region $\mathrm{P}_{ \pm 34 \%}$ from it. Fig. 6 shows the results of the clustering excess of CR arriving from radio galaxies obtained from the simulation.

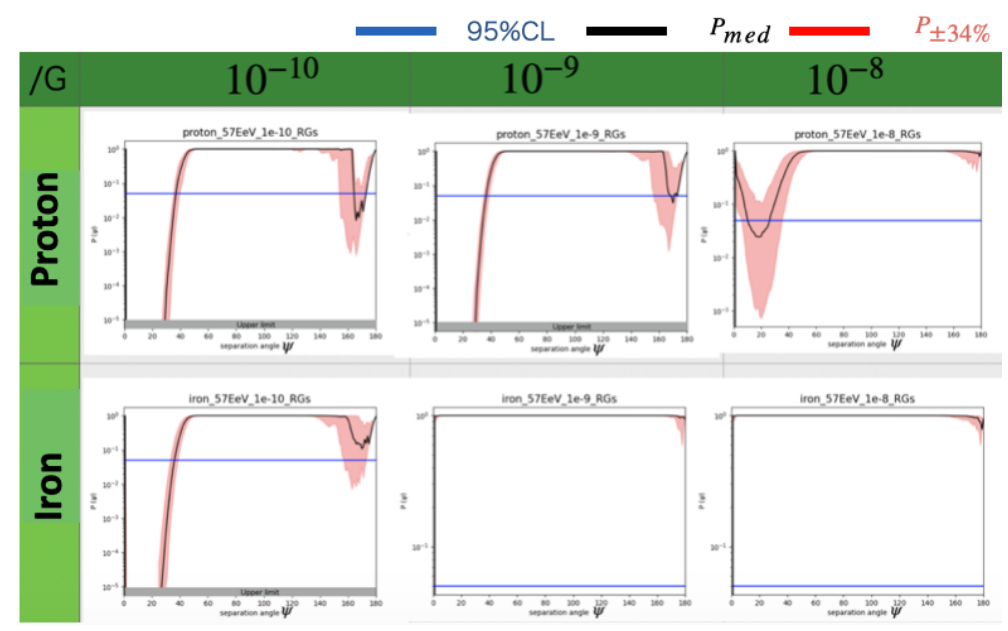

Figure 6: The horizontal axis shows Separation angle $\psi$, and the vertical axis shows the excess of clustering in the direction of cosmic ray arrival, $\mathrm{P}(\psi) .3$ different magnetic field strengths are assumed, the upper one for protons and the lower one for iron. The black line shows the median of $\mathrm{P}(\psi)$ obtained by comparing $10^{5}$ isotropic MC simulations with 100 data sets generated from MC simulations of the same number of events observed in the TA experiment. The red line is the statistical error of $\mathrm{P}(\psi)$ and shows the region $68 \%$ from the median. The blue line shows the $95 \%$ confidence level.

The black line shows the median of $\mathrm{P}(\psi)$ obtained by comparing $10^{5}$ isotropic MC simulations with 100 data sets generated from MC simulations of the same number of events observed in the TA experiment. The red line is the statistical error of $\mathrm{P}(\psi)$ and shows the region $68 \%$ from the median. The blue line shows the 95\% confidence interval. The results in Fig. 6, when assuming three types of magnetic fields and two types of nuclei, each show a characteristic structure. When the deflection is large, the distribution is almost isotropic. When the deflection is small, the change in the distribution is very small. When the magnetic field is assumed to be $10^{-9}$, there is a large difference between the proton and iron distributions. The scale of iron distribution is different only. It is also below the $95 \%$ confidence level, indicating that it is consistent with isotropic distribution. It can be said the iron distribution is almost isotropic due to the large deflection angle. Also, when 
the magnetic field is small, there is almost no difference in the $68 \%$ region. This means that the arrival direction is mostly scattered around the source due to the small deflection angle. Finally, show summary and discussion.

\section{Summary and Discussion}

We calculated the autocorrelation function of the expected CR arrival direction distribution from the RGs list. The results in Fig. 6, when assuming three types of magnetic fields and two types of nuclei, each show a characteristic structure. When the deflection is large, the distribution is almost isotropic. When the deflection is small, the change in the distribution is very small. In order to further confirm these results, we would like to determine the cosmic ray energy according to the energy spectrum of the source and to consider the energy loss distance by GZK suppression [5][15] for each particle species. We also hope to increase the number of data sets of isotropic MC and MC direction of arrival distributions, so that We may be able to check the minimum value of $\mathrm{P}(\psi)$. And we would like to use this analysis method for various new data sets of different source types.

\section{References}

[1] T. Abu-Zayyad et al., Nucl. Instrum. Meth. A 689, 87 (2012)

[2] K. Kawata et al., PoS(ICRC2019)310

[3] E. Kido et al, PoS(ICRC2019) 312

[4] Abu-Zayyad, et al.,The Astrophysical Journal, 757:26(11pp), 2012 September 20

[5] O. Deligny1, K. Kawata, and P. Tinyakov, PTEP 2017, 12A104

[6] P. Tinyakov et al., PoS(ICRC2015) 326

[7] J.P.Rachen, et al., ICRC2019, PoS 396

[8] B.Eichmann, JCAP 05 (2019) 009

[9] Hyesung Kang, jorg P. Rachen, Peter L. Biermann, Mon.Not.Roy.Astron.Soc. 286 (1997) 257

[10] Todor Stanev, Peter L. Biermann, Jeremy Lloyd-Evans, jorg P. Rachen and Alan A. Waston, astro-ph, 11 sep 1995

[11] M.Nagano, et al.,Rev. Mod. Phys., Vol. 72, No. 3, July 2000

[12] J. D. Bray and A. M. M. Scaife, ApJ, 8613

[13] A. D. ANGELIS et al. , ApJ, arXiv:0711.3346v3

[14] Paul Sommers, ApJ, arXiv:0004016v1

[15] D. Harari et al., Astrophysics, JCAP0611, 012, 2006 


\section{Full Authors List: Telescope Array Collaboration}

R.U. Abbasi ${ }^{1,2}$, T. Abu-Zayyad ${ }^{1,2}$, M. Allen ${ }^{2}$, Y. Arai ${ }^{3}$, R. Arimura ${ }^{3}$, E. Barcikowski², J.W. Belz ${ }^{2}$, D.R. Bergman ${ }^{2}$, S.A. Blake ${ }^{2}$, I. Buckland ${ }^{2}$, R. $\mathrm{Cady}^{2}$, B.G. Cheon ${ }^{4}$, J. Chiba ${ }^{5}$, M. Chikawa ${ }^{6}$, T. Fujii ${ }^{7}$, K. Fujisue ${ }^{6}$, K. Fujita ${ }^{3}$, R. Fujiwara ${ }^{3}$, M. Fukushima ${ }^{6}$, R. Fukushima ${ }^{3}$, G. Furlich ${ }^{2}$, R. Gonzalez ${ }^{2}$, W. Hanlon ${ }^{2}$, M. Hayashi ${ }^{8}$, N. Hayashida ${ }^{9}$, K. Hibino ${ }^{9}$, R. Higuchi ${ }^{6}$, K. Honda $^{10}$, D. Ikeda ${ }^{9}$, T. Inadomi ${ }^{11}$, N. Inoue ${ }^{12}$, T. Ishii ${ }^{10}$, H. Ito $^{13}$, D. Ivanov ${ }^{2}$, H. Iwakura ${ }^{11}$, A. Iwasaki ${ }^{3}$, H.M. Jeong ${ }^{14}$, S. Jeong ${ }^{14}$, C.C.H. Jui ${ }^{2}$, K. Kadota ${ }^{15}$, F. Kakimoto ${ }^{9}$, O. Kalashev ${ }^{16}$, K. Kasahara ${ }^{17}$, S. Kasami ${ }^{18}$, H. Kawai ${ }^{19}$, S. Kawakami ${ }^{3}$, S. Kawana ${ }^{12}$, K. Kawata ${ }^{6}$, I. Kharuk ${ }^{16}$, E. Kido ${ }^{13}$, H.B. Kim ${ }^{4}$, J.H. Kim ${ }^{2}$, J.H. Kim², M.H. Kim ${ }^{14}$, S.W. Kim ${ }^{14}$, Y. Kimura ${ }^{3}$, S. Kishigami ${ }^{3}$, Y. Kubota ${ }^{11}$, S. Kurisu ${ }^{11}$, V. Kuzmin ${ }^{16^{*}}$, M. Kuznetsov ${ }^{16,20}$, Y.J. Kwon ${ }^{21}$, K.H. Lee ${ }^{14}$, B. Lubsandorzhiev ${ }^{16}$, J.P. Lundquist ${ }^{2,22}$, K. Machida ${ }^{10}$, H. Matsumiya ${ }^{3}$, T. Matsuyama $^{3}$, J.N. Matthews ${ }^{2}$, R. Mayta ${ }^{3}$, M. Minamino ${ }^{3}$, K. Mukai ${ }^{10}$, I. Myers ${ }^{2}$, S. Nagataki ${ }^{13}$, K. Nakai $^{3}$, R. Nakamura ${ }^{11}$, T. Nakamura ${ }^{23}$, T. Nakamura ${ }^{11}$, Y. Nakamura ${ }^{11}$, A. Nakazawa ${ }^{11}$, E. Nishio ${ }^{18}$, T. Nonaka ${ }^{6}$, H. Oda ${ }^{3}$, S. Ogio $^{3,24}$, M. Ohnishi ${ }^{6}$, H. Ohoka ${ }^{6}$, Y. Oku ${ }^{18}$, T. Okuda ${ }^{25}$, Y. Omura ${ }^{3}$, M. Ono ${ }^{13}$, R. Onogi ${ }^{3}$, A. Oshima ${ }^{3}$, S. Ozawa ${ }^{26}$, I.H. Park ${ }^{14}$, M. Potts ${ }^{2}$, M.S. Pshirkov ${ }^{16,27}$, J. Remington ${ }^{2}$, D.C. Rodriguez ${ }^{2}$, G.I. Rubtsov ${ }^{16}$, D. Ryu ${ }^{28}$, H. Sagawa ${ }^{6}$, R. Sahara ${ }^{3}$, Y. Saito ${ }^{11}$, N. Sakaki ${ }^{6}$, T. Sako ${ }^{6}$, N. Sakurai ${ }^{3}$, K. Sano ${ }^{11}$, K. Sato ${ }^{3}$, T. Seki ${ }^{11}$, K. Sekino ${ }^{6}$, P.D. Shah ${ }^{2}$, Y. Shibasaki ${ }^{11}$, F. Shibata ${ }^{10}$, N. Shibata ${ }^{18}$, T. Shibata ${ }^{6}$, H. Shimodaira ${ }^{6}$, B.K. Shin ${ }^{28}$, H.S. Shin ${ }^{6}$, D. Shinto ${ }^{18}$, J.D. Smith ${ }^{2}$, P. Sokolsky ${ }^{2}$, N. Sone ${ }^{11}$, B.T. Stokes ${ }^{2}$, T.A. Stroman ${ }^{2}$, Y. Takagi ${ }^{3}$, Y. Takahashi ${ }^{3}$, M. Takamura ${ }^{5}$, M. Takeda ${ }^{6}$, R. Takeishi ${ }^{6}$, A. Taketa ${ }^{29}$, M. Takita ${ }^{6}$, Y. Tameda ${ }^{18}$, H. Tanaka ${ }^{3}$, K. Tanaka ${ }^{30}$, M. Tanaka ${ }^{31}$, Y. Tanoue ${ }^{3}$, S.B. Thomas ${ }^{2}$, G.B. Thomson ${ }^{2}$, P. Tinyakov ${ }^{16,20}$ I. Tkachev $^{16}$, H. Tokuno ${ }^{32}$, T. Tomida ${ }^{11}$, S. Troitsky ${ }^{16}$, R. Tsuda ${ }^{3}$, Y. Tsunesada ${ }^{3,24}$, Y. Uchihori ${ }^{33}$, S. Udo ${ }^{9}$, T. Uehama ${ }^{11}$, F. Urban ${ }^{34}$, T. Wong ${ }^{2}$, K. Yada $^{6}$, M. Yamamoto ${ }^{11}$, K. Yamazaki ${ }^{9}$, J. Yang ${ }^{35}$, K. Yashiro ${ }^{5}$, F. Yoshida ${ }^{18}$, Y. Yoshioka ${ }^{11}$, Y. Zhezher ${ }^{6,16}$, and Z. Zundel ${ }^{2}$

${ }^{1}$ Department of Physics, Loyola University Chicago, Chicago, Illinois, USA

${ }^{2}$ High Energy Astrophysics Institute and Department of Physics and Astronomy, University of Utah, Salt Lake City, Utah, USA

${ }^{3}$ Graduate School of Science, Osaka City University, Osaka, Osaka, Japan

${ }^{4}$ Department of Physics and The Research Institute of Natural Science, Hanyang University, Seongdong-gu, Seoul, Korea

${ }^{5}$ Department of Physics, Tokyo University of Science, Noda, Chiba, Japan

${ }^{6}$ Institute for Cosmic Ray Research, University of Tokyo, Kashiwa, Chiba, Japan

7 The Hakubi Center for Advanced Research and Graduate School of Science, Kyoto University, KitashirakawaOiwakecho, Sakyo-ku, Kyoto, Japan

${ }^{8}$ Information Engineering Graduate School of Science and Technology, Shinshu University, Nagano, Nagano, Japan

${ }^{9}$ Faculty of Engineering, Kanagawa University, Yokohama, Kanagawa, Japan

${ }^{10}$ Interdisciplinary Graduate School of Medicine and Engineering, University of Yamanashi, Kofu, Yamanashi, Japan

${ }^{11}$ Academic Assembly School of Science and Technology Institute of Engineering, Shinshu University, Nagano, Nagano, Japan

${ }^{12}$ The Graduate School of Science and Engineering, Saitama University, Saitama, Saitama, Japan

${ }^{13}$ Astrophysical Big Bang Laboratory, RIKEN, Wako, Saitama, Japan

${ }^{14}$ Department of Physics, SungKyunKwan University, Jang-an-gu, Suwon, Korea

${ }^{15}$ Department of Physics, Tokyo City University, Setagaya-ku, Tokyo, Japan

${ }^{16}$ Institute for Nuclear Research of the Russian Academy of Sciences, Moscow, Russia

${ }^{17}$ Faculty of Systems Engineering and Science, Shibaura Institute of Technology, Minato-ku, Tokyo, Japan 
${ }^{18}$ Department of Engineering Science, Faculty of Engineering, Osaka Electro-Communication University, Neyagawashi, Osaka, Japan

${ }^{19}$ Department of Physics, Chiba University, Chiba, Chiba, Japan

${ }^{20}$ Service de Physique Théorique, Université Libre de Bruxelles, Brussels, Belgium

${ }^{21}$ Department of Physics, Yonsei University, Seodaemun-gu, Seoul, Korea

22 Center for Astrophysics and Cosmology, University of Nova Gorica, Nova Gorica, Slovenia

${ }^{23}$ Faculty of Science, Kochi University, Kochi, Kochi, Japan

${ }^{24}$ Nambu Yoichiro Institute of Theoretical and Experimental Physics, Osaka City University, Osaka, Osaka, Japan

${ }^{25}$ Department of Physical Sciences, Ritsumeikan University, Kusatsu, Shiga, Japan

${ }^{26}$ Quantum ICT Advanced Development Center, National Institute for Information and Communications Technology, Koganei, Tokyo, Japan

27 Sternberg Astronomical Institute, Moscow M.V. Lomonosov State University, Moscow, Russia

${ }^{28}$ Department of Physics, School of Natural Sciences, Ulsan National Institute of Science and Technology, UNIST-gil, Ulsan, Korea

${ }^{29}$ Earthquake Research Institute, University of Tokyo, Bunkyo-ku, Tokyo, Japan

${ }^{30}$ Graduate School of Information Sciences, Hiroshima City University, Hiroshima, Hiroshima, Japan

${ }^{31}$ Institute of Particle and Nuclear Studies, KEK, Tsukuba, Ibaraki, Japan

${ }^{32}$ Graduate School of Science and Engineering, Tokyo Institute of Technology, Meguro, Tokyo, Japan

${ }^{33}$ Department of Research Planning and Promotion, Quantum Medical Science Directorate, National Institutes for Quantum and Radiological Science and Technology, Chiba, Chiba, Japan

${ }^{34}$ CEICO, Institute of Physics, Czech Academy of Sciences, Prague, Czech Republic

${ }^{35}$ Department of Physics and Institute for the Early Universe, Ewha Womans University, Seodaaemun-gu, Seoul, Korea

\section{Acknowledgements:}

The Telescope Array experiment is supported by the Japan Society for the Promotion of Science(JSPS) through Grants-in-Aid for Priority Area 431, for Specially Promoted Research JP21000002, for Scientific Research (S) JP19104006, for Specially Promoted Research JP15H05693, for Scientific Research (S) JP15H05741 and JP19H05607, for Science Research (A) JP18H03705, for Young Scientists (A) JPH26707011, and for Fostering Joint International Research (B) JP19KK0074, by the joint research program of the Institute for Cosmic Ray Research (ICRR), The University of Tokyo; by the Pioneering Program of RIKEN for the Evolution of Matter in the Universe (r-EMU); by the U.S. National Science Foundation awards PHY-1404495, PHY-1404502, PHY-1607727, PHY-1712517, PHY-1806797 and PHY-2012934; by the National Research Foundation of Korea (2017K1A4A3015188, 2020R1A2C1008230, \& 2020R1A2C2102800) ; by the Ministry of Science and Higher Education of the Russian Federation under the contract 075-15-2020-778, RFBR grant 20-02-00625a (INR), IISN project No. 4.4501.18, and Belgian Science Policy under IUAP VII/37 (ULB). This work was partially supported by the grants of The joint research program of the Institute for Space-Earth Environmental Research, Nagoya University and Inter-University Research Program of the Institute for Cosmic Ray Research of University of Tokyo. The foundations of Dr. Ezekiel R. and Edna Wattis Dumke, Willard L. Eccles, and George S. and Dolores Doré Eccles all helped with generous donations. The State of Utah supported the project through its Economic Development Board, and the University of Utah through the Office of the Vice President for Research. The experimental site became available through the cooperation of the Utah School and Institutional Trust Lands Administration (SITLA), U.S. Bureau of Land Management (BLM),

\footnotetext{
${ }^{*}$ Deceased
} 
and the U.S. Air Force. We appreciate the assistance of the State of Utah and Fillmore offices of the BLM in crafting the Plan of Development for the site. Patrick A. Shea assisted the collaboration with valuable advice and supported the collaboration's efforts. The people and the officials of Millard County, Utah have been a source of steadfast and warm support for our work which we greatly appreciate. We are indebted to the Millard County Road Department for their efforts to maintain and clear the roads which get us to our sites. We gratefully acknowledge the contribution from the technical staffs of our home institutions. An allocation of computer time from the Center for High Performance Computing at the University of Utah is gratefully acknowledged. 